\title{
Les douleurs en rhumatologie
}

\section{Rheumatic Pain}

\author{
A. Coutaux \\ (C) Lavoisier SAS 2015
}

Près de $50 \%$ les patients souffrant de douleurs chroniques adressés en consultation de la douleur le sont pour des douleurs en rapport avec une maladie rhumatologique, le plus souvent mécanique (arthrose des membres inférieurs, lombalgie) ou séquellaire de la chirurgie (lombosciatalgie sur rachis opéré, échec de remplacement prothétique...). Peu de patients souffrant de rhumatisme inflammatoire sont adressés en dehors d'avis diagnostiques concernant des tableaux douloureux diffus évoquant une spondyloarthropathie sans cependant en remplir les critères diagnostiques, et donc très proches de la fibromyalgie. Le monde de la rhumatologie a connu ces dernières années des avancées spectaculaires dans la connaissance de la physiopathologie de nombreux rhumatismes inflammatoires chroniques mais également de pathologies jusqu'alors un peu délaissées comme l'arthrose. De nouvelles cibles thérapeutiques ont pu être définies, permettant le développement de nouvelles thérapeutiques telles les biothérapies dirigées contre le TNF- $\alpha$ qui ont bouleversé le devenir des patients atteints de polyarthrite rhumatoïde notamment.

Ce numéro dédié à la rhumatologie permettra au lecteur de bénéficier des avancées faites dans la connaissance de l'arthrose, dans le diagnostic positif de la fibromylagie, et non plus par défaut, mais également de mieux appréhender les lombalgies chroniques en replaçant l'examen clinique en première ligne de toute prise en charge à la recherche de « lombalgies » peut-être curables. Les bénéfices attendus des cures thermales sont rediscutés au vu des données de la littérature pour permettre de mieux en parler avec nos patients. La démarche d'éducation thérapeutique fait partie intégrante du traitement de nombreuses maladies chroniques et les rhumatologues l'ont intégrée depuis plusieurs années dans la prise en charge des patients souffrant de rhumatismes inflammatoires chroniques sous biothérapies, mais également dans l'ostéoporose et la lombalgie. Cette démarche se développe progressivement à destination des patients douloureux chroniques, et pourrait bénéficier de l'« avance » prise par nos confrères et de leurs difficultés, notamment dans l'évaluation de ces programmes. Il est souvent reproché aux structures de prise en charge de la douleur de peu évaluer leurs pratiques, ici encore la démarche d'évaluation de l'efficacité thérapeutique des anti-TNF- $\alpha$ à partir des patient reported-outcome pourrait nous aider à construire des outils pratiques centrés sur les besoins des patients. Enfin, les antiTNF- $\alpha$ pourraient avoir une place dans l'arsenal thérapeutique de certains tableaux douloureux chroniques fréquemment rencontrés dans les structures d'évaluation et de traitement de la douleur.

À la lecture de ces articles, nous espérons que vous allez pouvoir enrichir votre pratique grâce à l'acquisition de nouvelles compétences cliniques mais également théoriques, ouvrant le champ à de nouveaux questionnements toujours pertinents pour nos patients.

\footnotetext{
A. Coutaux $(\bowtie)$

Consultation d'évaluation et de traitement de la douleur, service du Pr B. Fautrel, hôpital de la Pitié-Salpêtrière, 47 boulevard de l'Hôpital, F-75013 Paris, France

e-mail : anne.coutaux@psl.aphp.fr
} 